\title{
Research of SOA-based Data Exchange Platform
}

\author{
Qian-zhong Zhang \\ Department of Computer Science and Technolgy, \\ Cheng-Dong College of Northeast Agricultural University \\ Harbin, 150025,China \\ icantalk@126.com
}

\begin{abstract}
Complexity and versatility of problems encountered in the process in order to improve the traditional data exchange platform, "Proxy + server "loosely coupled mode data exchange platform system, system using a messaging service to solve complex interactive control information in the data exchange process. Solve the data exchange process in a variety of data transmission and efficient mutual use of data transmission services. System implementation process draws on SOA architecture Thought, part of the using of the core technology of SOA, Clear hierarchy of the system. Has good versatility and maintainability, greatly reduce the difficulty of system development.
\end{abstract}

Keywords-component; Data Exchange Platform;ServiceOriented Architecture;Message Service;Data Services;Enterprise bus Introduction

\section{INTRODUCTION}

With the information construction is booming, the major companies and universities, the number of applications running huge, more frequent exchanges between the various application systems, increasingly urgent demand for data exchange. Data Exchange Platform is an integrated service platform for companies and universities within a variety of data exchange and sharing needs, Construction and implementation of data exchange platform, Will be able to solve the data sharing and integration issues between applications, For enterprises and universities within the application system to provide a global view of the data, the global data permissions to view and improve the data exchange service, Companies and universities within many applications so as to solve the problem of information silos "federal model" (loose coupling between an application system model),Various data resources to achieve interoperability between applications.

\section{RESEARCH ON DATA EXCHANGE PlatFORM}

\section{A. Overview of Data Exchange Platform}

domestic and international data exchange system old design thinking after, The DEP system uses server + agentbased loosely coupled architecture model, Structure (shown in Figure I) the following features: DEP is a distributed open network system. It is constituted by a plurality of application software in a DEP Server DEP Agent, Applications in data exchange as an interface to DEP Agent,
Through interactive information exchange with DEP Server news and data.

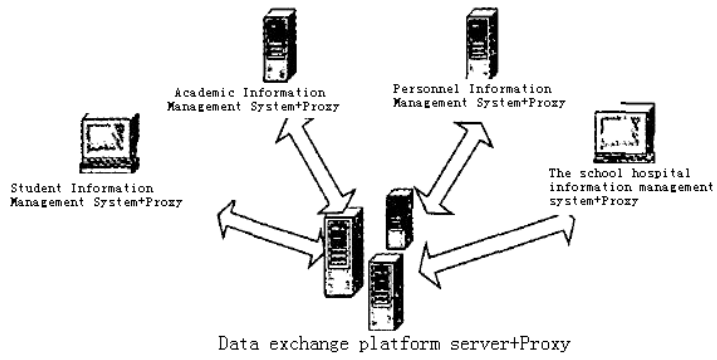

FiguareI. SOA-based data exchange platform applications

The DEP Server DEP's core management center, It is in the middle of all the information channel. DEP Server's main function is to provide data exchange service for each of the applications of the data exchange system, Including: messaging services, the exchange of data upload /download, Services, exchange data access services, exchange data subscription service.

DEP Agent is an important part of DEP, It is an interactive bridge between the DEP and various application systems. DEP Agent's primary function is to capture the event of the exchange of data of the application system to exchange data in accordance with the DEP data transmission specification, Then interact through messages with DEP Server, Control exchange data through the correct transmission of the DEP Server, Ensuring the data exchange process is completed correctly. DEP Agent integrated within the application system can be used as a logic member, Is also available as a Independent entity attached to the outside of the applications.

\section{B. Service mode of data exchange platform}

DEP provides data exchange service model including Publish Subscribe request "and" response ".

1) "Publish Subscribe "mode

The mode is the data exchange service provider (source data system, The same below) will exchange data DEP Agent service release to DEP Server, Demand-side data exchange service (application system of the source data, the same below), Subscribe to the corresponding DEP Server service by DEP Agent, The service provider will automatically changes the source data is sent to the 
subscription service the demand side related services; The mode is the service provider to initiate data exchange mode.

2) "Request Response" mode

The model is the demand side of the data exchange service implementation of the relevant request to DEP Server DEP Agent for switched data services, DEP Server through interaction and data exchange services provider for results, Feedback in response to the demand side of the data exchange; The mode is the service needs to initiate the data exchange mode.

\section{Data exchange platform services architecture}

The DEP service architecture can be designed according to the characteristics of the DEP service model: The between DEP Agent and DEP Server to complete the entire process of data exchange via the data exchange protocol, Data transmission services and messaging services to exchange data transmission and data exchange process flow control.

DEP in the data exchange process is constituted by two parts, update the exchange of data and control messages interaction, Data flow and message flow. In traditional data exchange system, data flow and message flow is indiscriminate, Either a data packet includes both the exchange of data, and also contains the corresponding contents of the message, Parsing need to parse the message at the same time and data, thus making the system complexity is very high, Both separately design and processing procedures, respectively, according to their characteristics, the system can effectively reduce The implementation complexity.

Data transmission services, the main function is to achieve high-speed data transmission path, Ensure the exchange of data timeliness, Reliability and consistency, and supports a variety of data transmission mode. Data exchange platform through a unified and standard data transfer protocols, Exchange of standardized data transmission in the between DEP Server DEP Agent, Data transmission services will be based on the size of the amount of data transmitted using different transmission modes, in order to achieve efficient transmission of the data stream.

Message service main function is to ensure the safety and reliability of data exchange, Party in the data exchange process, the exchange of data as well as data exchange platform needs to be implemented through the message transfer control of the data exchange process. Including the data update notification message mechanism, the exchange of data request, the confirmation of data receiving, and data transmission errors, error correction, etc. Data exchange platform to develop and implement a unified and standardized data exchange messaging protocols, The application system must communicate through the standard message protocols and data exchange platforms and other applications, to control the entire process of the data exchange.

\section{SOA-BASED DATA EXCHANGE PLATFORM IMPLEMENTATION}

\section{A. SOA-based system architecture thinking}

DEP system structure characteristics, service model and service architecture above shows that: DEP is a loosely coupled architecture-based data exchange system; Abstract DEP in accordance with the characteristics of its functions, and to standardize the form of services provided; The need to use a DEP in the news services and data transmission services to support heterogeneous technology based on uniform standards.

According to the above characteristics of DEP, System implementation process should be loosely coupled architecture model, In order to improve the versatility of DEP, The system should be based on uniform technical standards. SOA software architecture based on ideas to meet the system demand of loosely coupled and based on uniform technical standards, SOA architecture ideas introduced in the implementation process of the DEP system.

In a hierarchical design, DEP uses a hierarchical division of SOA thinking, The whole system is divided into six levels: business process layer, service layer, component layer, enterprise service bus layer and quality of service and safety management. After the hierarchical division, is conducive to building the DEP service-oriented architecture, the DEP system more standardized and universal.

Widespread adoption of SOA technology standards on the use of technology, DEP, Message module using unified messaging service JNIS to achieve, Web Service data transmission module cross-language platform to achieve data transmission; Integration and management of service registration and query module uses the enterprise service bus technology.

\section{B. SOA-based data exchange platform implementation}

The DEP system from the perspective deployment two parts divided into the DEP Server and DEP Agent, While from the perspective of system implementation, Can be six levels, eight core modules, Following from the hierarchy and module structures are introduced to achieve the DEP system.

\section{1) DEP system hierarchy:}

DEP hierarchy reference SOA-based system design ideas, the schematic diagram shown in Figure II .

From the hierarchy, the main function of the DEP will be composed of the following four:

Level to achieve: Service layer: (1)This layer is the most important layer of the system, The DEP all core services are concentrated in this layer, the layer component layer of functional components to build function DEP need different services. The so-called services, is a functional entity that has a unified and standardized service interface, service scheduling mode, perform a specific function. Most core services layer two services: news services and data services (data sharing service, data forwarding services). (2) Component layers: Component layer the DEP basic package into different components in the form, Such as data acquisition, data storage, and news subscriptions and the like, for the service of the upper particle moderate 
components. (3)Enterprise service bus, ESB: DEP service registration and management services provided query. There is little need to specify ESB SOA architecture system also assumed the function of the message service, In the DEP system specifically in the design process, the message service because the service provided in the entire data exchange process, so the news service was placed under the service layer. (4)Quality management, security management and monitoring layer: Log of the layer mainly provides services and authentication services, Provide services in the DEP process safety management and quality of service (such as: to ensure the robustness of the service).

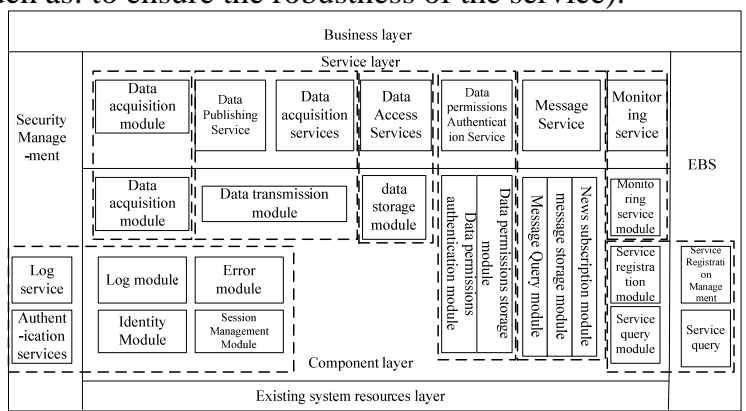

FiguareII. SOA-based data exchange platform level

The above four layers to achieve the main body of the DEP, DEP in the specific arrangements for the use of the process in addition to the above four layers also need the following two levels to convergence between the DEP and the business system. (5)Business layer: The layer using the package to build a process for data exchange between different applications by DEP Agent DEP Server between good variety of data exchange service. (6)Existing system resources layer, The layer with the already existing information systems, Such as customer information management system, employee information management system., These systems will interact with the component layer, take advantage of the functionality of the existing system as far as possible, to reduce the amount of system development.

2) The DEP system module structure:

From the module structure, 8 core modules to achieve the main function of the DEP system, These modules according to the division level is decomposed into multiple sub-modules, each sub-divided into modules according to its characteristics of the different levels of DEP, The eight core module name and their main function is as follows:

- Message module: The main processing interoperability between DEP Agent and DEP Server control information. The protocol control the entire process of data exchange through the for unified specification data exchange messages.

- Data transmission module: Mainly deal with the problem of data transfer between DEP Agent and DEP Server. Through a unified and standard data transfer protocols, exchange data transmission between the DEP Server and DEP Agent, based on uniform data standards.

- Service monitoring module: The process of data exchange service monitoring, to ensure the full implementation of the service, while in abnormal circumstances exception handling, to ensure the correctness of data exchange service.

- Data storage module: DEP systems require a variety of mechanisms store process data, the need for access to the important data in the source data and destination data exchange process. For store-and-forward type, And only temporary in DEP Server and DEP Agent storage: Shared data to the data the demand side data exchange platform, Long-term storage in the DEP Server-side.

- Data privileges storage and data access authentication module: Data authority in order to ensure the storage data security and consistency, through the verification of the data permissions restrictions, System can control the fine-grained (field level) control to data access, User role allows only the appropriate permissions to access.

- Service registration and query module: Service registration and query module provides management capabilities for data exchange related services such as registration and cancellation, Data exchange services that allow agents to release and cancel the proxy, For ease of maintenance, the service module provides services query function.

- Authentication and session management module: In order to ensure the DEP system security, system by connection to the server-side proxy authentication server authentication module.

- Error handling and logging module: In order to guarantee the DEP system fault tolerance, System through error handling and logging two modules the DEP case an error occurs during operation, the system for processing and recording, DEP can quickly restore the system in case of an error, logging also provides important operating information in the process of using the system.

DEP system for the analysis and design of the two dimensions of the system level and module complete realization of ideas, Clear interface between the various levels of the system, standardized access method, the technology standard. Especially because of the the DEP system in the typical data exchange service better abstract are concentrated to the service layer, DEP system specific implementation process, Only need to adjust the business logic layer implementations that can meet the actual demand of data exchange platform system, which can greatly reduce the software designed to adjust the workload requirements change.

\section{USING THE TEMPLATE}

This article by abstract data exchange platform messaging service and data service, DEP system loosely coupled architecture. The DEP systems through server + agent architecture model, And learn from the SOA architecture hierarchical implementation of data exchange platform, Applications to establish a loosely coupled data exchange system: separation through multiple levels of services, components, enterprise bus, All levels to focus on their own priorities; The message in the data exchange, data transmission, data storage, authentication, data authority control and other functions; At the same time due to the low 
coupling between the data exchange services and can be combined usability, Easy modification of the data exchange process and the reconstruction of the data exchange system. Currently, the system has the practical application of the implementation of data exchange platform project in Tsinghua University. Practice has proved that the DEP system effectively reduce the complexity of the data exchange system implementation process, has good versatility and maintainability.

\section{REFERENCES}

[1] Zi-yao Wang, Jun-jie Nan and Zi-hui Duan "SOA Core technology and applications ",Bei-jing, Publishing House of Electronics Industry,2008,PP.34-39
[2] Xin Shui,Yun-long Zhu,Lin Nan,” Design and implementation of supply chain management platform based on SOA", Technology and industry,2012 pp.78-82

[3] Kezunovic M.Ownership of Data and the Need for Information Exchange.Belgrade,Yugoslavia:Balkan Power Conference,2002, pp.234-239

[4] Erradi A,Mahesbwari P,wsBus.QoS-Aware Middleware for Reliable Web Services Interaction,The 2005 IEEE Internationnal Conference on e-Technology,e-Commerce ande e-Service.Hong Kong;IEEE Computer Society,2005, pp.634-639

[5] Yu M,Tableb-Bendiab A,Reilly D.A polyarchical middleware for selfregenerative invaocation of multi-standard ubiquitous services,IEEE Intermational Conference on Web Services,Shanghai,IEEE Computer Society,2004,pp.410-417 